\title{
Nuclear osteopontin-c is a prognostic breast cancer marker
}

\author{
K Zduniak ${ }^{1}$, P Ziolkowski ${ }^{1}$, C Ahlin ${ }^{2}$, A Agrawal ${ }^{3}$, S Agrawal ${ }^{3}$, C Blomqvist ${ }^{4}$, M-L Fjällskog ${ }^{4}$ and G F Weber ${ }^{\star}, 5$ \\ ${ }^{1}$ Department of Pathology, Wroclaw Medical University, 50-367 Wroclaw, Poland; ${ }^{2}$ Department of Oncology, Örebro University \\ Hospital, SE-70185 Örebro, Sweden; ${ }^{3}$ Department of General and Oncological Surgery, Wroclaw Medical University, 50-367 \\ Wroclaw, Poland; ${ }^{4}$ Department of Radiology, Oncology and Radiation Sciences, Uppsala University, 75105 Uppsala, Sweden and \\ ${ }^{5}$ College of Pharmacy, University of Cincinnati Academic Health Center, Cincinnati, OH 45267, USA
}

Background: Although Osteopontin has been known as a marker for cancer progression, the elevated production of this cytokine is not specific for cancer. We have identified the splice variant Osteopontin-c as being absent from healthy tissue but associated with about $75 \%$ of breast cancer cases. However, in previous studies of Osteopontin-c, follow-up information was not available.

Methods: Here we have analysed 671 patients, comprising a cohort of 291 paraffin blocks plus a population-based case-control study of 380 arrayed breast tumor tissues.

Results: We find that high staining intensity of nuclear Osteopontin-c is strongly associated with mortality in patients with early breast cancer. Cytosolic staining for exon 4, reflective of Osteopontin-a and -b also predicts poor outcome. By contrast, total Osteopontin does not correlate with prognosis. These diverse assessments of Osteopontin also do not correlate with each other, suggesting distinct expression patterns for the variant forms. Consistent with its role in tumor progression, not tumor initiation, Osteopontin-c is not correlated with proliferation markers (Ki-67, cyclin A, cyclin B, cyclin E and cyclin D), neither is it correlated with ER, PR or HER2.

Conclusions: The addition of Osteopontin-c immunohistochemistry to standard pathology work-ups may have prognostic benefit in early breast cancer diagnosis.

The goals of cancer therapy are to eliminate the transformed cells, limit the risk of local recurrence and minimise the prospect of invasive spread. In breast cancer, it is based on surgery (lumpectomy or mastectomy) and adjuvant treatment (hormonal therapy, chemotherapy and radiation). Treatment choices are difficult to make for individual patients, because there are no predictors for their specific progression risk. While an early diagnosis is critical for the successful management of the disease, the detection of early-stage lesions poses the challenge of accurately assessing the risk of breast cancer recurrence and death. Available diagnostic techniques involve a biopsy where samples of tissue are taken to confirm or eliminate the presence of cancer cells by histopathological examination. While this procedure is a standard at present, it does not detect invasive potential. The prognostic/ predictive factors most commonly used to select patients for adjuvant therapy are age, lymph node status, tumor size, hormone receptors, histologic grade, proliferation (Ki-67) and human epidermal growth factor receptor 2 (HER2). The oestrogen receptor status is used for prediction of endocrine responsiveness, $\mathrm{Ki}-67$ to assess the likelihood of response to chemotherapy and HER2 status to determine the need of HER2-directed therapy. The practiced regimen can be improved if reliable molecular markers are identified to assess the aggressiveness of a tumor at an early stage. By including such an evaluation, physicians will be better able to determine appropriate treatment options for their patients.

Osteopontin has been associated with the progression of numerous types of cancer (Weber et al, 2010, 2011; Weber, 2011), including those of the breast. The full-length form of the molecule (Osteopontin-a) physiologically acts as a $\mathrm{TH}_{1}$ cytokine that may be secreted by macrophages and T-lymphocytes and is

*Correspondence: Dr GF Weber; E-mail: georg.weber@uc.edu

Received 10 September 2014; revised 4 December 2014; accepted 12 December 2014; published online 27 January 2015

(c) 2015 Cancer Research UK. All rights reserved 0007-0920/15 
elevated in the blood during immune responses (Ashkar et al, 2000). Further, the osteopontin gene (SPP1) is oestrogen responsive (Craig and Denhardt, 1991) and Osteopontin-a is secreted during lactation (Senger et al, 1989), limiting its value as a breast cancer marker. We have identified the splice variant Osteopontin-c (He et al, 2006) to be selectively present in specific cancers including those of the breast (Mirza et al, 2008; Sullivan et al, 2009; Tilli et al, 2011), but not in healthy tissue, and to serve as a marker for tumor grade (Mirza et al, 2008; Hartung and Weber, 2013). These studies were done by immunohistochemistry or by real-time RT-PCR on the cancer tissues or in patient blood. A prognostic role for Osteopontin-c RNA in breast cancer has been suggested independently (Patani et al, 2008a,b). Here, we study the value of Osteopontin-c immunohistochemistry as a prognostic indicator in breast cancer.

\section{MATERIALS AND METHODS}

Patients. The study has two source populations, comprising 671 patients. Part 1 contained 291 Polish patients who presented between 1995 and 2008 (allowing the assessment of 5-year survival). All cases refer to invasive ductal carcinoma, grades 1, 2 and 3, with subtypes including two mucinous and five tubular carcinomas. Information about the patients was received from the Department of General and Oncological Surgery, Wroclaw and from the Division of Oncological Surgery, Walbrzych, Poland. The inclusion criteria were size of tumor not $>50 \mathrm{~mm}$ and no adjuvant chemotherapy. For all patients, who met these criteria, paraffin blocks were available for evaluation. The data comprised also information about pathological TNM, BRCA1 status, HER2, ER and PR status, and family history (other cases of invasive breast carcinoma in the family). The study was approved by the local ethics committee in Wroclaw, Poland. Part 2 was a defined collection of women diagnosed with breast cancer in the UppsalaÖrebro region in 1993-2004. Information about the patients was derived from the Uppsala-Örebro Breast Cancer Register, which is a population-based clinical database with coverage of over $98 \%$. Inclusion criteria were tumor size not $>50 \mathrm{~mm}$, no lymph node metastases and no adjuvant chemotherapy. Within this cohort, cases were defined as women who died from breast cancer. About 240 cases were identified using the regional quality register for breast cancer and the national register for causes of death. Controls were women alive at the time of the corresponding case's death. For each identified case, one control was randomly selected. About 50 cases and corresponding controls were excluded from the study for not fulfilling the inclusion criteria after reviewing data from patient files and pathology reports or because of missing tumor blocks: 26 patients had new/contralateral or locally advanced breast cancer, in 12 patients no paraffin blocks were found, 6 patients had non-breast cancer death, 4 patients had distant metastases at diagnosis, 1 patient received adjuvant chemotherapy and 1 patient

Table 1. Patient characteristics

\begin{tabular}{|c|c|c|c|c|c|c|c|c|}
\hline & \multicolumn{2}{|c|}{ Polish cohort } & \multicolumn{6}{|c|}{ Swedish cohort } \\
\hline & Case & & Controls & & Case & & Controls & \\
\hline & $n$ & $\%$ & $n$ & $\%$ & $n$ & $\%$ & $n$ & $\%$ \\
\hline \multicolumn{9}{|l|}{$T$} \\
\hline 0 & 0 & 0.0 & 1 & 0.5 & - & - & - & - \\
\hline 1 & 31 & 32.0 & 100 & 51.5 & - & - & - & - \\
\hline 2 & 41 & 42.3 & 60 & 30.9 & - & - & - & - \\
\hline 3 & 6 & 6.2 & 2 & 1.0 & - & - & - & - \\
\hline \multicolumn{9}{|l|}{$\mathbf{N}$} \\
\hline 0 & 35 & 36.1 & 94 & 48.5 & - & - & - & - \\
\hline 1 & 14 & 14.4 & 26 & 13.4 & - & - & - & - \\
\hline 2 & 16 & 16.5 & 26 & 13.4 & - & - & - & - \\
\hline 3 & 13 & 13.4 & 17 & 8.8 & - & - & - & - \\
\hline \multicolumn{9}{|l|}{ HER2 } \\
\hline- & 44 & 45.4 & 100 & 51.5 & 158 & 83.2 & 161 & 84.7 \\
\hline+ & 15 & 15.5 & 51 & 26.3 & 18 & 9.5 & 13 & 6.8 \\
\hline \multicolumn{9}{|l|}{ PR } \\
\hline- & 42 & 43.3 & 93 & 47.9 & 108 & 56.8 & 60 & 31.6 \\
\hline+ & 30 & 30.9 & 65 & 33.5 & 73 & 38.4 & 127 & 66.8 \\
\hline \multicolumn{9}{|l|}{ ER } \\
\hline- & 35 & 36.1 & 77 & 39.7 & 79 & 41.6 & 41 & 21.6 \\
\hline+ & 37 & 38.1 & 81 & 41.8 & 103 & 54.2 & 147 & 77.4 \\
\hline \multicolumn{9}{|c|}{ BRCA1 } \\
\hline Normal & 25 & 25.8 & 68 & 35.1 & - & - & - & - \\
\hline Mutant & 13 & 13.4 & 51 & 26.3 & - & - & - & - \\
\hline \multicolumn{9}{|c|}{ Familial } \\
\hline No & 24 & 24.7 & 82 & 42.3 & - & - & - & - \\
\hline Yes & 15 & 15.5 & 59 & 30.4 & - & - & - & - \\
\hline \multicolumn{9}{|c|}{ Hormonal therapy } \\
\hline Yes & - & - & - & - & 53 & 27.9 & 48 & 25.3 \\
\hline \multicolumn{9}{|c|}{ Adjuvant radiotherapy } \\
\hline Yes & - & - & - & - & 101 & 53.2 & 116 & 61.1 \\
\hline
\end{tabular}


A

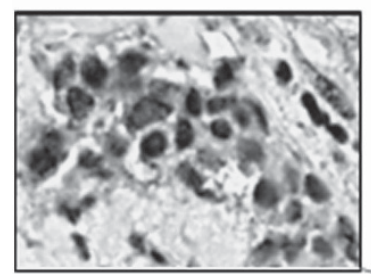

hOPNc grade 1

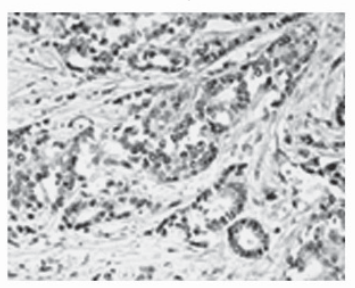

hOPNc grade 1, 1+

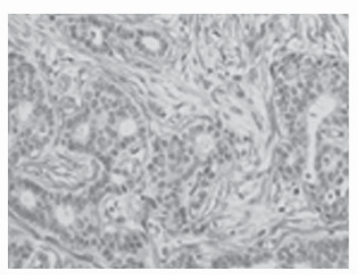

Exon 4 grade 2

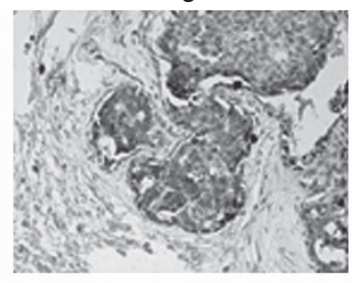

hOPNc grade 2

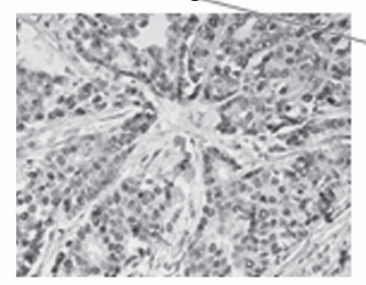

hOPNc grade $2,2+$

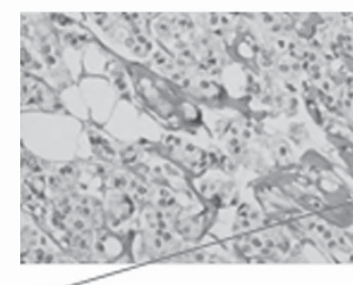

panOPN grade $2,2+$

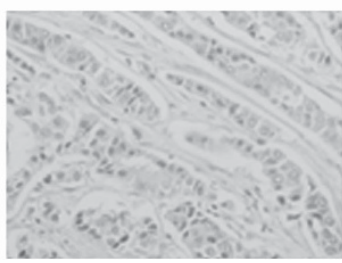

Exon 4 grade 3

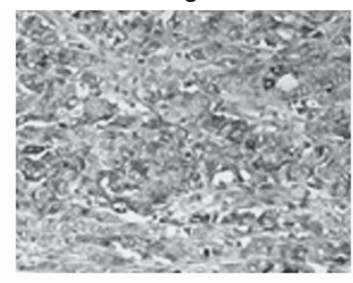

hOPNc grade 3

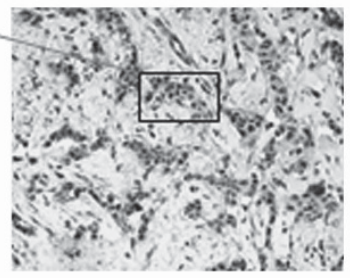

hOPNc grade 3, 3+

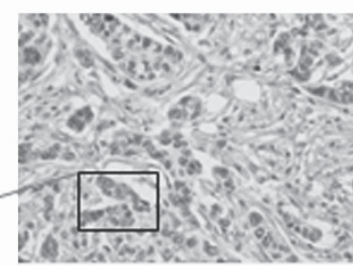

panOPN grade $3,3+$

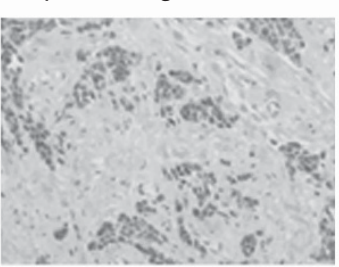

B

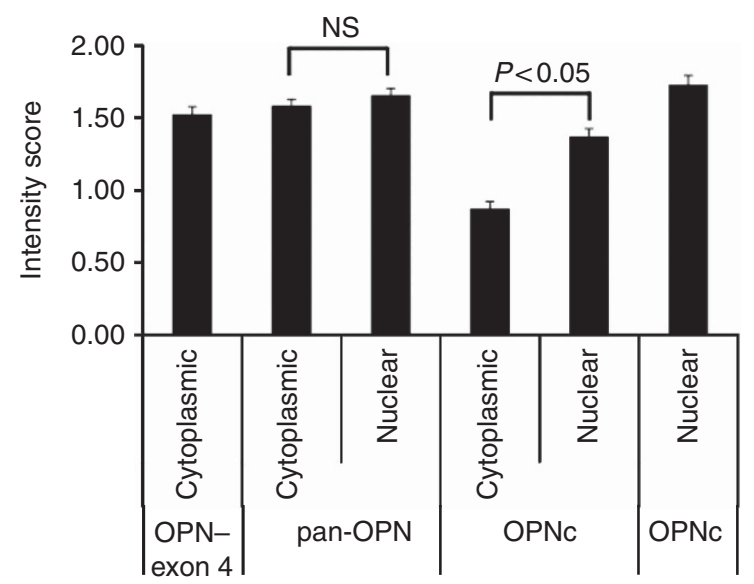

Figure 1. Osteopontin staining. (A) (top row) Cytoplasmic staining for Osteopontin-exon 4 in invasive ductal carcinomas (histopathological grades 2-3, and staining intensity 2-3) from the Polish cohort. (Second row) Nuclear staining of Osteopontin-c in invasive ductal carcinomas (histopathological grades 1-3, staining intensity 1-3) from the Polish cohort. The insert in the top row (left) shows a zoomed-in picture of the grade 3 staining. (Third row) Anti-Osteopontin-C staining of invasive ductal carcinomas (histopathological grades 1-3, staining intensity 1-3) from the Swedish cohort. The insert in the bottom row (left) shows a zoomed-in picture of the grade 3 staining. (bottom row) Anti-pan-Osteopontin staining in invasive ductal carcinomas from the Swedish cohort. For all pictures, counterstaining with hematoxilin was performed and the original magnification was $\times 200$. (B) Mean values and s.e. of the immunohistochemistry intensity scores for pan-Osteopontin, Osteopontin-c and Osteopontin-exon 4 are shown (for clarity, per cent positivity is not shown). The results for the Swedish cohort and Polish cohort (far left and right bars in the graph) are shown separately. Osteopontin-exon 4 is exclusively cytoplasmic, Osteopontin-c is predominantly nuclear and panOsteopontin distributes in both compartments. The differences between nuclear and cytoplasmic staining intensity were assessed by $t$-test and a $P$-value $<0.05$ was considered significant. NS, not significantly different. 
had no breast surgery performed. The study was approved by the local ethics committee in Uppsala, Sweden.

Immunohistochemistry. The antibodies used in this study, after blocking in $2 \%$ donkey serum, were O-17 (IBL America, Minneapolis, MN, USA), anti-hOPNc IgY (Gallus Immunotech, Fergus, ON, Canada) and LF161 (Dr Larry Fisher, NIH). The polyclonal rabbit antibody $\mathrm{O}-17$ recognises an epitope upstream of the splice junctions and thus is common to all three forms of Osteopontin (anti-pan-Osteopontin). It was used at dilution $1: 100$. The Osteopontin-c (IgY) antibody recognises the Osteopontin-c splice junction and detects the molecule in immunohistochemistry (Mirza et al, 2008; Sullivan et al, 2009, 2011; Tilli et al, 2012; Pang et al, 2013; Zhang et al, 2013; Ortiz-Martínez et al, 2014). It was diluted at $1: 500$ to $1: 700$. The polyclonal rabbit antibody LF161 for selectively staining exon 4 (present in Osteopontin-a and -b) was used at $1: 1000$. For each antibody, the tissues were scored for intensity (maximum intensity of

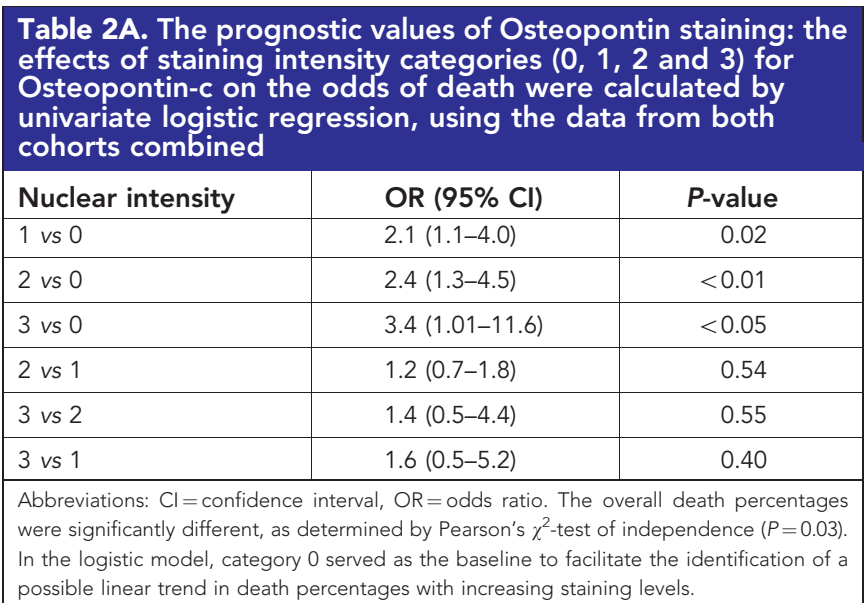

Table 2B. Prognostic values of Osteopontin staining: predictive value of OPNc, Osteopontin-exon 4 (exon 4) or pan-OPN immunohistochemistry for patient survival, split up according to nucl.int., nucl.per., cyt.int. and cyt.per.

\begin{tabular}{|c|c|c|c|c|}
\hline & & & \multicolumn{2}{|c|}{$95 \% \mathrm{Cl}$} \\
\hline & Odds ratio & Significance & Lower & Upper \\
\hline \multicolumn{5}{|l|}{ Polish cohort } \\
\hline Exon 4 cyt.int. & 1.88 & $<0.001$ & 1.40 & $2.56^{\star}$ \\
\hline Exon 4 cyt.per. & 0.99 & 0.540 & 0.98 & 1.01 \\
\hline OPNc nucl.int. & 1.52 & 0.001 & 1.19 & $1.95^{\star}$ \\
\hline OPNc nucl.per. & 1.00 & 0.684 & 0.99 & 1.01 \\
\hline \multicolumn{5}{|l|}{ Swedish cohort } \\
\hline Pan-OPN nucl.int. & 0.72 & 0.066 & 0.51 & 1.02 \\
\hline Pan-OPN nucl.per. & 1.00 & 0.732 & 0.99 & 1.01 \\
\hline Pan-OPN cyt.int. & 1.00 & 0.997 & 0.70 & 1.43 \\
\hline Pan-OPN cyt.per. & 0.99 & 0.059 & 0.98 & 1.00 \\
\hline OPNc cyt.int. & 1.38 & 0.058 & 0.99 & 1.93 \\
\hline OPNc cyt.per. & 0.99 & 0.374 & 0.98 & 1.01 \\
\hline OPNc nucl.int. & 1.47 & 0.007 & 1.11 & $1.94^{\star}$ \\
\hline OPNc nucl.per. & 1.00 & 0.393 & 0.99 & 1.00 \\
\hline \multicolumn{5}{|l|}{ Combined } \\
\hline OPNc nucl.int. & 1.50 & $<0.001$ & 1.25 & $1.81^{\star}$ \\
\hline OPNc nucl.per. & 1.00 & 0.290 & 1.00 & 1.01 \\
\hline \multicolumn{5}{|c|}{$\begin{array}{l}\text { Abbreviations: } \mathrm{Cl}=\text { confidence interval; cyt.int. = cytosolic intensity; cyt.per. = cytosolic per } \\
\text { cent positivity; nucl.int. = nuclear intensity; nucl.per. = nuclear per cent positivity; } \\
\text { pan-OPN=pan-Osteopontin. The two study populations (Polish } n=291 \text {, Swedish } n=380 \text { ) } \\
\text { are evaluated separately (top two sections). The combined analysis (bottom section) is } \\
\text { adjusted for group (the regression model of survival indicated that there was a group effect; } \\
\text { the overlapping confidence intervals in the calculations for OPN nucl.int. corroborate its } \\
\text { prognostic usefulness). Significant values are marked with an asterisk. }\end{array}$} \\
\hline
\end{tabular}

the sample $0,1,2$ or 3 ) and per cent positivity (low= $5(0 \%-10 \%)$, medium $=30(10 \%-50 \%)$, high $=75(50 \%-100 \%))$, separately for nuclei and cytoplasm (Dabbs, 2010). In the Polish cohort, for each antibody a formalin fixed and paraffin-embedded biopsy specimen from cancer tissue was cut on a microtome in $5 \mu \mathrm{m}$ slices. All microscopic slides were independently evaluated by two pathologists and in the rare cases of discrepant initial scores a final score was agreed after discussion. In the Swedish cohort, all tissues were represented twice on the arrays. There was a good correlation between the scoring results from the two biopsies, specifically for the intensity scores. For the Polish cohort, the EnVision kit from Dako (Carpinteria, CA, USA) was used as a detecting system in a Dako stainer. For the Swedish cohort, rehydrated sections of paraffin-embedded tissues were developed in Ventana's (Tucson, AZ, USA) Benchmark XT stainer using their CC1 (heated EDTA) antigen retrieval solution. For visualisation, after incubation with biotinylated, species-specific, donkey-raised secondary antibodies and avidin-conjugated peroxidase, DAB (brown) and hematoxylin were used.

Statistics. The primary method for addressing the study purposes was logistic repression. The odds of death were evaluated with respect to pathology scores and clinicopathological variables.

\begin{tabular}{|c|c|c|c|c|c|}
\hline \multicolumn{6}{|c|}{$\begin{array}{l}\text { Table 2C. Prognostic values of Osteopontin staining: } \\
\text { multivariate analysis of the Polish cohort for cytosolic and } \\
\text { nuclear Osteopontin-c intensity, as well as Osteopontin-exon } \\
4 \text { cytoplasmic intensity and patient survival }\end{array}$} \\
\hline & \multicolumn{5}{|c|}{$95 \% \mathrm{Cl}$} \\
\hline & $\begin{array}{l}\text { Odds } \\
\text { ratio }\end{array}$ & $P$-value & Lower & Upper & AIC \\
\hline OPNc nucl.int. & 2.13 & $<0.001$ & 1.51 & 3.08 & 224.0 \\
\hline Her2 & 0.67 & 0.26 & 0.33 & 1.32 & - \\
\hline OPNc nucl.int. & 1.57 & $<0.01$ & 1.19 & 2.09 & 270.9 \\
\hline ER & 0.94 & 0.83 & 0.53 & 1.66 & - \\
\hline OPNc nucl.int. & 1.56 & $<0.01$ & 1.19 & 2.08 & 271.0 \\
\hline PR & 1.02 & 0.95 & 0.57 & 1.80 & - \\
\hline OPNc nucl.int. & 1.43 & 0.013 & 1.09 & 1.91 & 278.4 \\
\hline Tumor size & 2.27 & $<0.01$ & 1.40 & 3.75 & - \\
\hline OPNc nucl.int. & 1.46 & $<0.01$ & 1.10 & 1.94 & 286.4 \\
\hline Lymph node & 1.19 & 0.18 & 0.92 & 1.52 & - \\
\hline OPNc nucl.int. & 1.43 & $<0.01$ & 1.11 & 1.85 & 324.2 \\
\hline Tumor grade & 2.64 & $<0.001$ & 1.40 & 3.75 & - \\
\hline Exon 4 cyt.int. & 2.15 & $<0.001$ & 1.47 & 3.24 & 228.8 \\
\hline Her2 & 0.55 & 0.1 & 0.26 & 1.10 & - \\
\hline Exon 4 cyt.int. & 1.82 & $<0.001$ & 1.30 & 2.60 & 269.6 \\
\hline ER & 1.03 & 0.92 & 0.58 & 1.82 & - \\
\hline Exon 4 cyt.int. & 1.82 & $<0.001$ & 1.30 & 2.60 & 269.6 \\
\hline PR & 0.99 & 0.99 & 0.56 & 1.77 & - \\
\hline Exon 4 cyt.int. & 1.78 & $<0.01$ & 1.25 & 2.58 & 275.1 \\
\hline Tumor size & 2.11 & $<0.01$ & 1.29 & 3.53 & - \\
\hline Exon 4 cyt.int. & 1.90 & $<0.001$ & 1.34 & 2.74 & 280.7 \\
\hline Lymph node & 1.21 & 0.15 & 0.94 & 1.55 & - \\
\hline Exon 4 cyt.int. & 1.83 & $<0.001$ & 1.33 & 2.55 & 318.3 \\
\hline Tumor grade & 2.51 & $<0.001$ & 1.68 & 3.83 & \\
\hline \multicolumn{6}{|c|}{$\begin{array}{l}\text { Abbreviations: } \mathrm{AlC}=\text { Akaike Information Criterion (a measure of the relative quality of a } \\
\text { statistical model applied to the data set); } \mathrm{Cl}=\text { confidence interval; cyt.int. = cytosolic } \\
\text { intensity; } E R=\text { oestrogen receptor; } \mathrm{HER} 2=\text { human epidermal growth factor receptor } 2 \\
\text { nucl.int. = nuclear intensity; } \mathrm{PR}=\text { progesterone receptor. For each immunohistochemical } \\
\text { readout, the numbers are presented in the order of lowest to highest AIC. The two study } \\
\text { cohorts are evaluated separately. }\end{array}$} \\
\hline
\end{tabular}


Odds ratios estimate the odds of death for a one-unit increase in the independent variable. Each cohort was analysed separately, the data were combined for both cohorts when appropriate. First, standard logistic regressions of the pathology scores were performed combining data from both cohorts, modelled continuously to detect a linear trend in the odds of death with respect to increasing score levels. Unadjusted odds ratios and $95 \%$ confidence intervals were calculated. Logistic regressions were also analysed to investigate the effects of the components of pathological scores on the odds of death. Each cohort was analysed separately. The nuclear Osteopontin-c staining values for both cohorts were evaluated for combined data, after a cohort indicator variable was added to the model. The biomarkers Osteopontin-c and Osteopontin-exon 4 were also analysed multivariately. Each model contained either Osteopontin-c or Osteopontin-exon 4 and each other biomarker (tumor size, tumor stage, lymph node involvement, HER2, progesterone receptor or oestrogen receptor), added one-at-a-time. Firth logistic regressions were performed to achieve model convergence, due to unbalanced and small numbers in the categories of some biomarkers. Firth's penalised likelihood is a method of addressing issues of separability and bias of parameter estimates (Firth, 1993). The optimum model was determined by minimisation of the Akaike Information Criterion (Akaike, 1974).

Correlations between Osteopontin-c and clinicopathological variables were assessed with Pearson's correlation test. Correlation coefficients of 0.1 to 0.3 are considered weak, $0.4-0.6$ is moderate correlation and $0.7-0.9$ is strong correlation. Analyses were performed using SAS, Version 9.4 (SAS Institute, Cary, NC, USA) and SPSS version 20. A $P$-value of 0.05 indicates statistical significance, unless stated otherwise.

\section{RESULTS}

Patient characteristics and immunohistochemistry. In the Polish cohort, 97 women died from breast cancer within 5 years, while 194 women were alive after this observation period. The average age was 60 years for non-survivors and 56 years for survivors.

Table 2D. Prognostic values of Osteopontin staining: multivariate analysis for the various measures of Osteopontin staining, focusing on nuclear Osteopontin-c intensity and cytoplasmic Osteopontin-exon 4 intensity as predictors of patient survival

\begin{tabular}{|c|c|c|c|c|c|}
\hline & \multicolumn{5}{|c|}{$95 \% \mathrm{Cl}$} \\
\hline & $\begin{array}{l}\text { Odds } \\
\text { ratio }\end{array}$ & $P$-value & Lower & Upper & AIC \\
\hline OPNc nucl.int. & 1.45 & 0.01 & 1.10 & 1.93 & 468.9 \\
\hline OPNc nucl.per. & 1.00 & 0.36 & 0.99 & 1.01 & - \\
\hline OPNc nucl.int. & 1.40 & 0.02 & 1.05 & 1.86 & 468.7 \\
\hline pan-OPN nucl.int. & 0.68 & 0.03 & 0.48 & 0.97 & - \\
\hline OPNc nucl.int. & 1.43 & 0.01 & 1.08 & 1.91 & 473.6 \\
\hline pan-OPN cyt.int. & 1.02 & 0.93 & 0.70 & 1.48 & - \\
\hline OPNc nucl.int. & 1.32 & 0.04 & 1.02 & 1.72 & 341.9 \\
\hline Exon 4 cyt.int. & 1.69 & 0.01 & 1.24 & 2.34 & - \\
\hline Exon 4 cyt.per. & 1.01 & 0.03 & 1.00 & 1.02 & 345.9 \\
\hline OPNc nucl.per. & 1.00 & 0.38 & 0.99 & 1.01 & - \\
\hline Exon 4 cyt.int. & 2.01 & $<0.01$ & 1.40 & 2.92 & 339.8 \\
\hline Exon 4 cyt.per. & 0.99 & 0.54 & 0.98 & 1.01 & \\
\hline $\begin{array}{l}\text { Abbreviations: } \mathrm{AIC}= \\
\text { cytosolic intensity; } \\
\text { nucl.per. = nuclear per }\end{array}$ & Inforn & on Criterio & $\begin{array}{l}\mathrm{Cl}=\text { conf } \\
\text { sitivity; }\end{array}$ & nce interve & $\begin{array}{l}\text { cyt.int. = } \\
\text { intensity }\end{array}$ \\
\hline
\end{tabular}

In the Swedish cohort, 190 women who died from breast cancer were defined as cases and 190 women alive at the time for the corresponding cases' deaths were defined as controls. The average age was 66 years for cases and 61 years for controls. Among all patients, $253(70.7 \%)$ had a tumor size $<20 \mathrm{~mm}$, while $105(29.3 \%)$ had tumours with diameters of $\geqslant 20 \mathrm{~mm}$. The average tumor size was $20 \mathrm{~mm}$ for cases and $16 \mathrm{~mm}$ for controls (Ahlin et al, 2009; Niméus-Malmström et al, 2010). All patients, comprising both cohorts, underwent surgery consisting of either modified radical mastectomy with axillary dissection, or conservative breast surgery with axillary lymph node dissection and post-operative irradiation of the breast (Table 1).

The anti-pan-Osteopontin antibody showed positive staining mostly in the cytoplasm. About 261 samples (68.7\%) had higher per cent positivity in the cytoplasm than in the nucleus, while $26(6.8 \%)$ samples had higher per cent positivity in the nucleus than in the cytoplasm. The staining intensity was comparable between the compartments, with 115 samples (30.3\%) displaying higher staining intensity in the cytoplasm, while 138 samples (36.3\%) had higher staining intensity in the nucleus; the intensity score was equal between cytoplasm and nucleus in all other specimens.
OPNc intensity

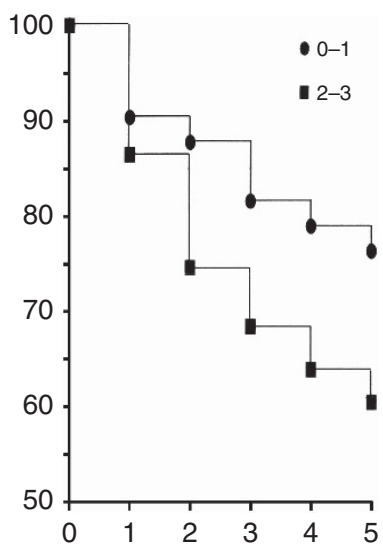

OPNc per cent

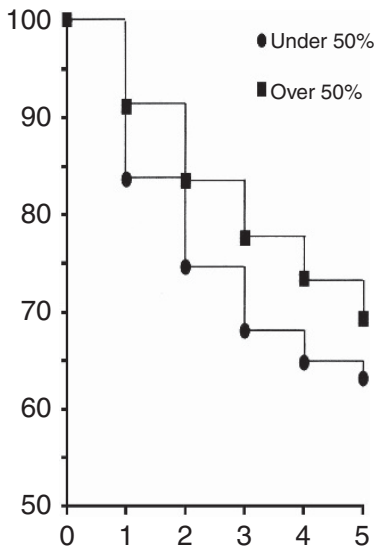

Exon 4 intensity

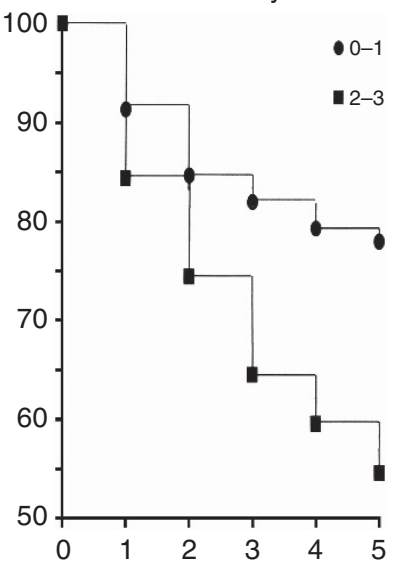

Exon 4 per cent

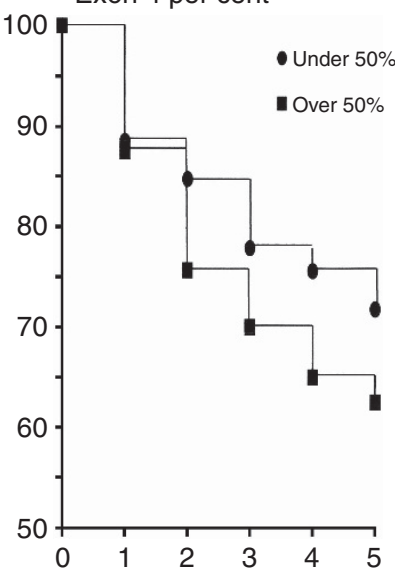

Figure 2. Kaplan-Meier survival curves. Shown are the survival curves of the Polish cohort for up to 5 years related to intensity or per cent positivity of Osteopontin-c or Osteopontin-exon 4. Of note, due to the design chosen for the Swedish breast cancer material (case-control design, where controls were sampled by incidence density sampling) it is not possible to calculate Kaplan-Meier curves. The mean time from diagnosis to recurrence for the Swedish patients was 1238 days for Osteopontin-c nuclear intensity 0, 1181 days for 0.5-1, 1130 days for $1.5-2$ and 1081 days for $2.5-3$. 
The anti-Osteopontin-exon 4 antibody, which recognises Osteopontin-a and -b, selectively stained the cytoplasm. Most tumours displayed Osteopontin-c predominantly in their nuclei. In the Swedish cohort, 239 samples (62.9\%) had higher staining intensity in the nucleus, while only 46 samples (12.1\%) had higher staining intensity in the cytoplasm (Figure 1).

Osteopontin variants and risk of breast cancer death. The correlation of staining intensities for Osteopontin-c with patient survival indicated that with higher levels of staining the odds of death increase linearly (Table 2A). Therefore, the simple and powerful linear model of correlation was subsequently applied. By univariate analysis, the staining intensity of Osteopontin-c in the nucleus is a negative prognostic indicator for patient survival in each cohort and in a combined evaluation adjusted for group. However, the per cent positivity of the staining shows essentially no prognostic association. The staining intensity, but not per cent positivity, for Osteopontin-a and -b in the cytoplasm (antiOsteopontin-exon-4 antibody) is prognostic for a high risk of death (Figure 2). By contrast, the anti-pan-Osteopontin antibody is not predictive, showing no significant correlations with prognosis (Table 2B).

We performed multivariate analyses on Osteopontin-c staining intensity or Osteopontin-exon 4 staining intensity, accounting for tumor size, lymph node involvement, tumor grade, HER2 status, progesterone receptor status and oestrogen receptor status. In both cases, the primary marker maintained significance at the 0.01 level, individually and combined. The best model, according to the

Table 3. Correlations between Osteopontin variant immunohistochemistry scores

\begin{tabular}{|c|c|c|c|c|c|c|c|}
\hline Polish cohort & & & $\begin{array}{c}\text { OPNc } \\
\text { nucl.per. }\end{array}$ & $\begin{array}{c}\text { OPNc } \\
\text { nucl.int. }\end{array}$ & $\begin{array}{l}\text { Exon } 4 \\
\text { cyt.per. }\end{array}$ & & \\
\hline \multicolumn{8}{|l|}{ Exon 4 Cyt.int. } \\
\hline $\begin{array}{l}\text { Correlation } \\
P \text {-value } \\
N\end{array}$ & & & $\begin{array}{l}0.21666 \\
\leq 0.001 \\
302\end{array}$ & $\begin{array}{l}0.40395 \\
\leq 0.001 \\
302\end{array}$ & $\begin{array}{l}0.61855 \\
\leq 0.001 \\
302\end{array}$ & $\begin{array}{l}- \\
- \\
-\end{array}$ & $\begin{array}{l}- \\
- \\
-\end{array}$ \\
\hline \multicolumn{8}{|l|}{ Exon 4 Cyt.per. } \\
\hline $\begin{array}{l}\text { Correlation } \\
P \text {-value } \\
N\end{array}$ & & & $\begin{array}{l}0.26245 \\
\leq 0.001 \\
302\end{array}$ & $\begin{array}{l}0.38367 \\
\leq 0.001 \\
302\end{array}$ & $\begin{array}{l}- \\
-\end{array}$ & $\begin{array}{l}- \\
-\end{array}$ & $\begin{array}{l}- \\
-\end{array}$ \\
\hline \multicolumn{8}{|l|}{ OPNc Nucl.int. } \\
\hline $\begin{array}{l}\text { Correlation } \\
P \text {-value } \\
N\end{array}$ & & & $\begin{array}{l}0.53334 \\
\leq 0.001 \\
302\end{array}$ & $\begin{array}{l}- \\
- \\
-\end{array}$ & $\begin{array}{l}- \\
- \\
-\end{array}$ & $\begin{array}{l}- \\
- \\
-\end{array}$ & $\begin{array}{l}- \\
- \\
-\end{array}$ \\
\hline Swedish cohort & OPN cyt.per. & OPN cyt.int. & $\begin{array}{c}\text { OPN } \\
\text { nucl.per. }\end{array}$ & $\begin{array}{c}\text { OPN } \\
\text { nucl.int. }\end{array}$ & $\begin{array}{c}\text { OPNc } \\
\text { cyt.per. }\end{array}$ & OPNc cyt.int. & $\begin{array}{c}\text { OPNc } \\
\text { nucl.per. }\end{array}$ \\
\hline \multicolumn{8}{|l|}{ OPNc Nucl.int. } \\
\hline $\begin{array}{l}\text { Pearson correlation } \\
\text { Sig. (two-tailed) } \\
N\end{array}$ & $\begin{array}{l}0.066 \\
0.218 \\
355\end{array}$ & $\begin{array}{c}0.078 \\
0.141 \\
355\end{array}$ & $\begin{array}{c}-0.053 \\
0.317 \\
355\end{array}$ & $\begin{array}{l}-0.121 \\
\frac{0.022}{355}\end{array}$ & $\begin{array}{c}0.015 \\
0.773 \\
358\end{array}$ & $\begin{array}{l}0.399 \\
0 \\
3 \overline{5} 7\end{array}$ & $\begin{array}{c}-0.002 \\
0.968 \\
358\end{array}$ \\
\hline \multicolumn{8}{|l|}{ OPNc Nucl.per. } \\
\hline $\begin{array}{l}\text { Pearson correlation } \\
\text { Sig. (two-tailed) } \\
N\end{array}$ & $\begin{array}{l}0.031 \\
0.566 \\
355\end{array}$ & $\begin{array}{l}0.066 \\
0.212 \\
355\end{array}$ & $\begin{array}{c}0.025 \\
0.635 \\
355\end{array}$ & $\begin{array}{c}-0.006 \\
0.912 \\
355\end{array}$ & $\begin{array}{l}0.166 \\
\frac{0.002}{358}\end{array}$ & $\begin{array}{c}0.13 \\
0.014 \\
357\end{array}$ & $\begin{array}{l}- \\
- \\
-\end{array}$ \\
\hline \multicolumn{8}{|l|}{ OPNc Cyt.int. } \\
\hline $\begin{array}{l}\text { Pearson correlation } \\
\text { Sig. (two-tailed) } \\
N\end{array}$ & $\begin{array}{l}-0.077 \\
0.147 \\
354\end{array}$ & $\begin{array}{l}0.26 \\
3 \frac{0}{54}\end{array}$ & $\begin{array}{c}-0.207 \\
\frac{0}{3 \overline{5}}\end{array}$ & $\begin{array}{l}0.137 \\
\frac{0.01}{354}\end{array}$ & $\begin{array}{l}-0.169 \\
\frac{0.001}{357}\end{array}$ & $\begin{array}{l}- \\
- \\
-\end{array}$ & $\begin{array}{l}- \\
- \\
-\end{array}$ \\
\hline \multicolumn{8}{|l|}{ OPNc Cyt.per. } \\
\hline $\begin{array}{l}\text { Pearson correlation } \\
\text { Sig. (two-tailed) } \\
N\end{array}$ & $\begin{array}{c}0.088 \\
0.097 \\
355\end{array}$ & $\begin{array}{l}0.018 \\
0.73 \\
355\end{array}$ & $\begin{array}{c}0.011 \\
0.838 \\
355\end{array}$ & $\begin{array}{c}-0.045 \\
0.395 \\
355\end{array}$ & $\begin{array}{l}- \\
- \\
-\end{array}$ & $\begin{array}{l}- \\
- \\
-\end{array}$ & $\begin{array}{l}- \\
- \\
-\end{array}$ \\
\hline \multicolumn{8}{|l|}{ OPN Nucl.int. } \\
\hline $\begin{array}{l}\text { Pearson correlation } \\
\text { Sig. (two-tailed) } \\
N\end{array}$ & $\begin{array}{l}0.007 \\
0.89 \\
366\end{array}$ & $\begin{array}{c}0.093 \\
0.076 \\
366\end{array}$ & $\begin{array}{c}-0.553 \\
\frac{0}{366}\end{array}$ & $\begin{array}{l}- \\
-\end{array}$ & $\begin{array}{l}- \\
- \\
-\end{array}$ & $\begin{array}{l}- \\
-\end{array}$ & $\begin{array}{l}- \\
-\end{array}$ \\
\hline \multicolumn{8}{|l|}{ OPN Nucl.per. } \\
\hline $\begin{array}{l}\text { Pearson correlation } \\
\text { Sig. (two-tailed) } \\
N\end{array}$ & $\begin{array}{l}0.104 \\
\frac{0.048}{366}\end{array}$ & $\begin{array}{c}-0.246 \\
\frac{0}{366}\end{array}$ & $\begin{array}{l}- \\
- \\
-\end{array}$ & $\begin{array}{l}- \\
- \\
-\end{array}$ & $\begin{array}{l}- \\
- \\
-\end{array}$ & $\begin{array}{l}- \\
- \\
-\end{array}$ & $\begin{array}{l}- \\
- \\
-\end{array}$ \\
\hline \multicolumn{8}{|l|}{ OPN Cyt.int. } \\
\hline $\begin{array}{l}\text { Pearson correlation } \\
\text { Sig. (two-tailed) } \\
N\end{array}$ & $\begin{array}{c}-0.405 \\
0 \\
3 \frac{0}{6} 6\end{array}$ & $\begin{array}{l}- \\
-\end{array}$ & $\begin{array}{l}- \\
- \\
-\end{array}$ & $\begin{array}{l}- \\
-\end{array}$ & $\begin{array}{l}- \\
-\end{array}$ & $\begin{array}{l}- \\
- \\
-\end{array}$ & $\begin{array}{l}- \\
-\end{array}$ \\
\hline \multicolumn{8}{|c|}{ 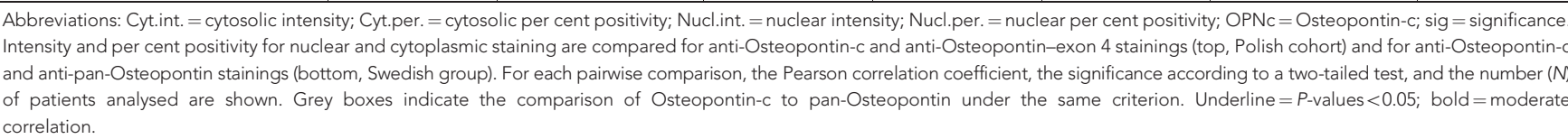 } \\
\hline
\end{tabular}


minimisation of the Akaike information content is the combination with HER2, followed by oestrogen receptor, progesterone receptor, tumor size, lymph node involvement and tumor grade (Table 2C). The multivariate analysis of the Swedish cohort yielded comparable results for Osteopontin-c nuclear staining intensity and oestrogen receptor (odds ratio 1.60, 95\% confidence interval $0.85-3.01, P$-value $=0.14$ ), tumor size (odds ratio 1.06 , $95 \%$ confidence interval $1.03-1.1, P$-value $=0.001)$ or tumor grade (odds ratio 1.63, 95\% confidence interval 1.07-2.48, $P$-value $=0.02$ ).

We further assessed whether the combination of the various readouts for Osteopontin staining would increase the prognostic value over the individual measurements. Only nuclear Osteopontin-c intensity plus cytoplasmic Osteopontin--exon-4 intensity resulted in significant $P$-values, 95\%-confidence intervals over 1.00 and a low Akaike information content compared with the other pairs of readouts tested (Table 2D). There may be a moderate gain in prognostication from this marker combination.

Correlations of Osteopontin forms to each other and to clinicopathological variables. As past immunohistochemistry studies have mostly used anti-pan-Osteopontin antibodies, it was important to compare the anti-Osteopontin-c (IgY) staining to the anti-pan-Osteopontin (O-17) staining. There was no correlation. However, cytoplasmic exon 4 staining intensity and nuclear Osteopontin-c staining intensity correlated moderately, consistent with the prognostic value of each marker for patient survival. For all antibodies, there was a moderate correlation between staining intensity and per cent positivity in the same compartment (Table 3).

From previous analyses of the cohorts under study, we had information on proliferation markers, as well as ER, PR and HER2. Expectedly, the proliferation marker Ki-67 correlated with cyclins, which are drivers of cell cycle progression, and with ER, which is a growth factor receptor. Likewise, the cyclins correlated among each other, and family history correlated with BRCA1 status. By contrast, there were no strong or moderate correlations between the abundance of Osteopontin-c or Osteopontin-exon 4 and any of these clinical variables (Table 4), suggesting that the Osteopontin splice variants provide readouts for tumor characteristics that are distinct from those associated with the main growth-regulating molecules.

Table 4. Osteopontin variant immunohistochemistry and clinicopathologic variables

\begin{tabular}{|c|c|c|c|c|c|c|c|}
\hline Polish cohort & ER status & PR status & Her2 status & BRCA1 status & $\begin{array}{l}\text { Family } \\
\text { history }\end{array}$ & $\begin{array}{c}\text { Tumor } \\
\text { stage } N\end{array}$ & $\begin{array}{c}\text { Tumor } \\
\text { stage } T\end{array}$ \\
\hline \multicolumn{8}{|l|}{ OPNc nucl.int. } \\
\hline Correlation & 0.07331 & 0.00433 & -0.00701 & 0.14347 & 0.05927 & 0.21711 & 0.14069 \\
\hline$P$-value & 0.263 & 0.9473 & 0.9183 & 0.073 & 0.4267 & 0.0006 & 0.0267 \\
\hline$N$ & 235 & 235 & 217 & 157 & 182 & $\overline{248}$ & $\overline{248}$ \\
\hline \multicolumn{8}{|c|}{ OPNc nucl.per. } \\
\hline Correlation & -0.07118 & -0.04898 & 0.07376 & 0.17555 & 0.08196 & 0.10875 & 0.08009 \\
\hline$P$-value & 0.27772 & 0.4549 & 0.2793 & 0.0279 & 0.2713 & 0.0874 & 0.2043 \\
\hline$N$ & 235 & 235 & 217 & 157 & 182 & 248 & 248 \\
\hline \multicolumn{8}{|l|}{ Exon 4 cyt.int. } \\
\hline Correlation & -0.03726 & 0.00904 & 0.11273 & 0.11684 & 0.07928 & 0.1472 & 0.21118 \\
\hline$P$-value & 0.5698 & 0.8904 & 0.0977 & 0.145 & 0.2874 & 0.0204 & 0.0008 \\
\hline N & 235 & 235 & 217 & 157 & 182 & $\overline{248}$ & $\overline{248}$ \\
\hline \multicolumn{8}{|c|}{ Exon 4 cyt.per. } \\
\hline Correlation & -0.00177 & -0.07063 & 0.14513 & 0.19729 & 0.08401 & 0.15886 & 0.13067 \\
\hline$P$-value & 0.9785 & 0.2809 & 0.0326 & 0.0133 & 0.2595 & 0.0122 & 0.0398 \\
\hline N & 235 & 235 & $\overline{217}$ & $\overline{157}$ & 182 & $\overline{248}$ & $\overline{248}$ \\
\hline \multicolumn{8}{|c|}{ Tumor stage $T$} \\
\hline Correlation & -0.05872 & -0.00674 & -0.04282 & -0.01138 & 0.08799 & 0.20622 & - \\
\hline$P$-value & 0.4137 & 0.9253 & 0.5682 & 0.8954 & 0.2701 & 0.001 & - \\
\hline$N$ & 196 & 196 & 180 & 136 & 159 & $\overline{248}$ & - \\
\hline \multicolumn{8}{|c|}{ Tumor stage $\mathbf{N}$} \\
\hline Correlation & -0.21586 & -0.18042 & -0.03603 & 0.01182 & -0.10732 & - & - \\
\hline$P$-value & 0.0024 & 0.0114 & 0.6311 & 0.8914 & 0.1781 & - & - \\
\hline N & $\frac{0.66}{196}$ & $\frac{196}{196}$ & 180 & 136 & 159 & - & - \\
\hline \multicolumn{8}{|l|}{ Family history } \\
\hline Correlation & 0.30712 & 0.29218 & 0.11734 & 0.43464 & - & - & - \\
\hline$P$-value & $<0.0001$ & 0.0002 & 0.1395 & $\leq 0.0001$ & - & - & - \\
\hline$N$ & 162 & $\overline{162}$ & 160 & 135 & - & - & - \\
\hline \multicolumn{8}{|l|}{ BRCA1 status } \\
\hline Correlation & -0.05872 & -0.00476 & 0.24007 & - & - & - & - \\
\hline$P$-value & 0.4137 & 0.9545 & 0.0044 & - & - & - & - \\
\hline$N$ & 196 & 146 & 139 & - & - & - & - \\
\hline \multicolumn{8}{|l|}{ Her2 status } \\
\hline Correlation & 0.05843 & 0.1501 & - & - & - & - & - \\
\hline$P$-value & 0.4257 & 0.0398 & - & - & - & - & - \\
\hline$N$ & 188 & $\overline{188}$ & - & - & - & - & - \\
\hline \multicolumn{8}{|l|}{ PR status } \\
\hline Correlation & -0.05872 & - & - & - & - & - & - \\
\hline$P$-value & 0.4137 & - & - & - & - & - & - \\
\hline$N$ & 196 & - & - & - & - & - & - \\
\hline
\end{tabular}




\begin{tabular}{|c|c|c|c|c|c|c|c|c|}
\hline Swedish cohort & PR status & ER status & Her2 status & Cyclin D & Cyclin E & Cyclin B & Cyclin A & $\mathrm{Ki}-67$ \\
\hline \multicolumn{9}{|l|}{ OPNc nucl.int. } \\
\hline $\begin{array}{l}\text { Pearson correlation } \\
\text { Sig. (two-tailed) } \\
N\end{array}$ & $\begin{array}{c}0.086 \\
0.109 \\
349\end{array}$ & $\begin{array}{c}0.008 \\
0.889 \\
350\end{array}$ & $\begin{array}{c}0.071 \\
0.194 \\
340\end{array}$ & $\begin{array}{l}0.23 \\
3 \frac{0}{4} 6\end{array}$ & $\begin{array}{c}0.057 \\
0.286 \\
354\end{array}$ & $\begin{array}{c}0.091 \\
0.087 \\
351\end{array}$ & $\begin{array}{c}0.098 \\
0.066 \\
349\end{array}$ & $\begin{array}{c}0.016 \\
0.773 \\
346\end{array}$ \\
\hline \multicolumn{9}{|l|}{ OPNc cyt.int. } \\
\hline $\begin{array}{l}\text { Pearson correlation } \\
\text { Sig. (two-tailed) } \\
N\end{array}$ & $\begin{array}{c}-0.036 \\
0.509 \\
348\end{array}$ & $\begin{array}{l}-0.124 \\
\frac{0.021}{349}\end{array}$ & $\begin{array}{c}-0.018 \\
0.737 \\
339\end{array}$ & $\begin{array}{c}0.17 \\
0.001 \\
345\end{array}$ & $\begin{array}{c}-0.008 \\
0.886 \\
353\end{array}$ & $\begin{array}{c}0.094 \\
0.079 \\
350\end{array}$ & $\begin{array}{c}0.03 \\
0.579 \\
348\end{array}$ & $\begin{array}{c}-0.006 \\
0.912 \\
345\end{array}$ \\
\hline \multicolumn{9}{|l|}{ Ki-67 } \\
\hline $\begin{array}{l}\text { Pearson correlation } \\
\text { Sig. (two-tailed) } \\
N\end{array}$ & $\begin{array}{c}0.362 \\
0 \\
3 \overline{3} 9\end{array}$ & $\begin{array}{c}0.404 \\
3 \overline{4} 0\end{array}$ & $\begin{array}{l}0.159 \\
\frac{0.004}{334}\end{array}$ & $\begin{array}{l}-0.185 \\
\frac{0.001}{340}\end{array}$ & $\begin{array}{c}0.467 \\
3 \frac{0}{4} 4\end{array}$ & $\begin{array}{c}0.602 \\
3 \frac{0}{4} 1\end{array}$ & $\begin{array}{c}0.674 \\
3 \frac{0}{4} 5\end{array}$ & $\begin{array}{l}- \\
- \\
-\end{array}$ \\
\hline \multicolumn{9}{|l|}{ Cyclin A } \\
\hline $\begin{array}{l}\text { Pearson correlation } \\
\text { Sig. (two-tailed) } \\
N\end{array}$ & $\begin{array}{c}0.382 \\
3 \frac{0}{4} 2\end{array}$ & $\begin{array}{c}0.425 \\
0 \\
3 \frac{4}{4} 3\end{array}$ & $\begin{array}{l}0.27 \\
3 \frac{0}{3} 5\end{array}$ & $\begin{array}{c}-0.139 \\
\frac{0.01}{341}\end{array}$ & $\begin{array}{c}0.518 \\
\frac{0}{34} 7\end{array}$ & $\begin{array}{l}0.69 \\
\frac{0}{34} 4\end{array}$ & $\begin{array}{l}- \\
-\end{array}$ & $\begin{array}{l}- \\
-\end{array}$ \\
\hline \multicolumn{9}{|l|}{ Cyclin B } \\
\hline $\begin{array}{l}\text { Pearson correlation } \\
\text { Sig. (two-tailed) } \\
N\end{array}$ & $\begin{array}{l}0.284 \\
3 \frac{0}{4} 2\end{array}$ & $\begin{array}{l}0.383 \\
3 \frac{0}{4} 3\end{array}$ & $\begin{array}{l}0.244 \\
3 \frac{0}{3} 6\end{array}$ & $\begin{array}{l}-0.107 \\
\frac{0.049}{341}\end{array}$ & $\begin{array}{c}0.526 \\
3 \frac{0}{50}\end{array}$ & $\begin{array}{l}- \\
-\end{array}$ & $\begin{array}{l}- \\
-\end{array}$ & $\begin{array}{l}- \\
-\end{array}$ \\
\hline \multicolumn{9}{|l|}{ Cyclin E } \\
\hline $\begin{array}{l}\text { Pearson correlation } \\
\text { Sig. (two-tailed) } \\
N\end{array}$ & $\begin{array}{l}0.289 \\
3 \frac{0}{4} 6\end{array}$ & $\begin{array}{l}0.391 \\
3 \overline{4} 7\end{array}$ & $\begin{array}{l}0.184 \\
0.001 \\
339\end{array}$ & $\begin{array}{l}-0.121 \\
\frac{0.025}{343}\end{array}$ & $\begin{array}{l}- \\
-\end{array}$ & $\begin{array}{l}- \\
-\end{array}$ & $\begin{array}{l}- \\
-\end{array}$ & $\begin{array}{l}- \\
-\end{array}$ \\
\hline \multicolumn{9}{|l|}{ Cyclin D } \\
\hline $\begin{array}{l}\text { Pearson correlation } \\
\text { Sig. (two-tailed) } \\
N\end{array}$ & $\begin{array}{c}-0.268 \\
3 \frac{0}{3} 8\end{array}$ & $\begin{array}{c}-0.406 \\
0 \\
3 \frac{0}{3} 9\end{array}$ & $\begin{array}{c}0.03 \\
0.582 \\
330\end{array}$ & $\begin{array}{l}- \\
-\end{array}$ & $\begin{array}{l}- \\
-\end{array}$ & $\begin{array}{l}- \\
-\end{array}$ & $\begin{array}{l}- \\
-\end{array}$ & $\begin{array}{l}- \\
-\end{array}$ \\
\hline \multicolumn{9}{|l|}{ Her2 status } \\
\hline $\begin{array}{l}\text { Pearson correlation } \\
\text { Sig. (two-tailed) } \\
N\end{array}$ & $\begin{array}{l}0.23 \\
3 \frac{0}{3} 3\end{array}$ & $\begin{array}{l}0.236 \\
3 \frac{0}{3} 4\end{array}$ & $\begin{array}{l}- \\
-\end{array}$ & $\begin{array}{l}- \\
-\end{array}$ & $\begin{array}{l}- \\
-\end{array}$ & $\begin{array}{l}- \\
-\end{array}$ & $\begin{array}{l}- \\
-\end{array}$ & $\begin{array}{l}- \\
-\end{array}$ \\
\hline \multicolumn{9}{|l|}{ ER status } \\
\hline $\begin{array}{l}\text { Pearson correlation } \\
\text { Sig. (two-tailed) } \\
N\end{array}$ & $\begin{array}{c}0.585 \\
0 \\
3 \frac{4}{4} 9\end{array}$ & $\begin{array}{l}- \\
-\end{array}$ & $\begin{array}{l}- \\
-\end{array}$ & $\begin{array}{l}- \\
-\end{array}$ & $\begin{array}{l}- \\
-\end{array}$ & $\begin{array}{l}- \\
-\end{array}$ & $\begin{array}{l}- \\
-\end{array}$ & $\begin{array}{l}- \\
-\end{array}$ \\
\hline \multicolumn{9}{|c|}{$\begin{array}{l}\text { Abbreviations: Cyt.int. = cytosolic intensity; Cyt.per. = cytosolic per cent positivity; ER=oestrogen receptor; Her } 2=\text { human epidermal growth factor receptor } 2 \text {; Nucl.int. = nuclear intensity; } \\
\text { Nucl.per. = nuclear per cent positivity; OPNc=Osteopontin-c; PR=progesterone receptor; sig=significance. Correlations are displayed between the immunohistochemistry scores for } \\
\text { Osteopontin-c or Osteopontin-exon } 4 \text { and molecular or clinical readouts. For each pairwise comparison, the Pearson correlation coefficient, the significance according to a two-tailed test and } \\
\text { the number }(N) \text { of patients analysed are shown. Tumor size was measured as the longest diameter, Ki- } 67 \text { and cyclin } A \text { were assessed as the maximum value of all punches in per cent. } \\
\text { Underline }=P \text {-values }<0.05 ; \text { bold = moderate correlation. }\end{array}$} \\
\hline
\end{tabular}

\section{DISCUSSION}

Our present finding that Osteopontin-c immunohistochemistry is a predictor of patient survival in breast cancer, which does not correlate with cyclins, receptor status or family predisposition, adds to previous reports that this splice form is an independent biomarker. In breast cancer tissue, Osteopontin-c (measured by real-time RT-PCR or immunohistochemistry) was present in over $75 \%$ of cases and served as an indicator of tumor grade (Mirza et al, 2008; Patani et al, 2008b; Pang et al, 2013). Pan-Osteopontin or Osteopontin-c showed no association with ER, PR or HER2, but Osteopontin-c was highly expressed in triple negative breast cancer (Mirza et al, 2008; Weber et al, 2011; Weber, 2011; Pang et al, 2013; Ortiz-Martínez et al, 2014). The multivariate analysis of this study (Table 2C) corroborates the prior observation that the diagnostic and prognostic values may be enhanced by combining Osteopontin-c with the receptor status of the cancer (Mirza et al, 2008). Osteopontin-c in breast cancers was reported to correlate with relapse (Pang et al, 2013; Ortiz-Martínez et al, 2014) or poor survival (Patani et al, 2008a,b). In the blood, breast carcinomas were associated with significantly higher levels of Osteopontin-c mRNA than carcinomas in situ. An elevation in Osteopontin-c RNA of 2 s.d. above the normal mean value detected a fraction of breast cancers, suggesting some heterogeneity within those types of tumours (Hartung and Weber, 2013). We conclude that patients with Osteopontin-c-positive breast cancers should consider adjuvant therapy to be mandatory.

The Osteopontin gene encodes for a signal sequence at the $\mathrm{N}$-terminal end of the molecule, and the protein is secreted. Consistently, the immunohistochemical signal for osteopontin is typically high in the perinuclear compartment, reflecting its transport through Golgi apparatus and endoplasmic reticulum for secretion. However, it has been described that Osteopontin may reach the nucleus of cells (Junaid et al, 2007) and we have previously observed, but not scored, nuclear staining for Osteopontin-c in breast cancer (Mirza et al, 2008). As the autocrine function of Osteopontin in breast cancer is inhibitable by neutralising antibodies (He et al, 2006), it is implied that the splice variant-c of the protein is secreted, taken back up and transported into the nucleus. The antibody to exon 4 detects mostly Osteopontin- $a$, as Osteopontin-b is barely expressed in breast cancer cells (Mirza et al, 2008). The anti-Osteopontin-exon-4 
antibody stains the tumor cell cytoplasm, suggesting that Osteopontin-a is not subject to nuclear uptake. The Osteopontin amino acid sequence reflects the presence of strong internalisation signals and adjacent pairs of basic amino acids, which is consistent with the possibility of nuclear import. The reason for the preferred nuclear localisation of Osteopontin-c compared with the preferred cytosolic staining for Osteopontin-exon 4 is unknown. It is not explained by the positions of the internalisation signals, which are common to all splice variants.

Osteopontin has been studied as a cancer progression marker for many years (Weber et al, 2010, 2011; Weber, 2011). However, the molecule has not found entry into clinical diagnostics. This may, in part, be due to the numerous post-translational modifications that create diverse forms of the Osteopontin protein, and in breast cancer to the oestrogen responsiveness and Osteopontin expression with the oestrous cycle. Improving current methods, antibodies are available to selectively detect Osteopontin splice variants. The abundance of exon 4 in the cytosolic compartment (distinct from the localisation of Osteopontin-c) effectively indicates prognosis. Osteopontin-c may be a more highly suitable disease progression marker as it is not subject to the background noise resulting from the physiologic production of Osteopontin in the breast (Osteopontin-c is absent from healthy cells). The amino acids in the immediate proximity of the Osteopontin-c splice junction are also not affected by the posttranslational structural variations that characterise the protein. Although Osteopontin splicing requires the synthesis of full-length RNA as an initial step and Osteopontin-c is never expressed without Osteopontin-a, the levels of these forms do not correlate (possibly because the rates of RNA synthesis and splicing are uncoupled, possibly due to differences in RNA or protein stability). Of note, the poor prognostic value of pan-Osteopontin staining together with the poor correlation between Osteopontin-c immunohistochemistry and pan-Osteopontin immunohistochemistry suggests that the distinct forms of the protein contribute variably to the pathophysiology of breast cancer, and that the measurement of total Osteopontin may even compromise the value of the progression biomarker compared with Osteopontin-c (or exon 4) alone.

Although a meta-analysis found the presence of Osteopontin, measured by various assays, to be predictive of poor survival of breast cancer patients (Weber et al, 2010), the literature describing the prognostic value of pan-Osteopontin immunohistochemistry on primary tumours is not consistent. When detected with antibody MBIII (Rudland et al, 2002) or MAb53 (Tuck et al, 1998), pan-Osteopontin staining is associated with poor patient survival. Staining with antibody mab1433 indicates an association with disease-free survival, but not with overall survival (Wang et al, 2008). By contrast, no association with survival is revealed with antibody LFMb-14 (Kim et al, 1998), and the same result is obtained here with antibody O-17. The apparently conflicting outcomes may be explained with the distinct epitopes recognised by the diverse anti-pan-Osteopontin antibodies. As the target molecule is subject to extensive post-translational processing, additional variables are introduced at specific domains and may affect prognostication. A focus on the splice variants largely eliminates this confounding component.

\section{ACKNOWLEDGEMENTS}

This research was supported by US DOD grants BC095225 and PR094070 to GFW. We are grateful to Dr Larry Fisher, NIH, for having generously provided the antibody LF161 (anti-Osteopontin-exon 4). Biostatistics support was provided by the Center for Clinical and Translational Science \& Training, Cincinnati.

\section{REFERENCES}

Ahlin C, Zhou W, Holmqvist M, Holmberg L, Nilsson C, Jirström K, Blomqvist C, Amini RM, Fjällskog ML (2009) Cyclin A is a proliferative marker with good prognostic value in node-negative breast cancer. Cancer Epidemiol Biomarkers Prev 18: 2501-2506.

Akaike H (1974) A new look at the statistical model identification. System identification and time-series analysis. IEEE Trans Automat Contr AC-19: 716-723.

Ashkar S, Weber GF, Panoutsakopoulou V, Sanchirico ME, Janssen M, Zawaideh S, Rittling S, Denhardt DT, Glimcher MJ, Cantor H (2000) Eta-1 (Osteopontin): An early component of type 1 (cell-mediated) immunity. Science 287: 860-864.

Craig AM, Denhardt DT (1991) The murine gene encoding secreted phosphoprotein 1 (osteopontin): promoter structure, activity, and induction in vivo by oestrogen and progesterone. Gene 100: 163-171.

Dabbs DJ (2010) Diagnostic Immunohistochemistry: Theranostic and Genomic Applications. 3rd edn. Philadelphia: Saunders Elsevier, pp 801-802.

Firth D (1993) Bias reduction of maximum likelihood estimates. Biometrika 80: $27-38$

Hartung F, Weber GF (2013) RNA Blood levels of osteopontin splice variants are cancer markers. SpringerPlus 2: 110.

He B, Mirza M, Weber GF (2006) An osteopontin splice variant induces anchorage independence in human breast cancer. Oncogene 25: 2192-2202.

Junaid A, Moon MC, Harding GE, Zahradka P (2007) Osteopontin localizes to the nucleus of 293 cells and associates with polo-like kinase-1. Am J Physiol Cell Physiol 292: C919-C926.

Kim YW, Park YK, Lee J, Ko SW, Yang MH (1998) Expression of osteopontin and osteonectin in breast cancer. J Korean Med Sci 13: 652-657.

Mirza M, Shaughnessy E, Hurley JK, Vanpatten KA, Pestano GA, He B, Weber GF (2008) Osteopontin-c is a selective marker for breast cancer. Int J Cancer 122: 889-897.

Niméus-Malmström E, Koliadi A, Ahlin C, Holmqvist M, Holmberg L, Amini RM, Jirström K, Wärnberg F, Blomqvist C, Fernö M, Fjällskog ML (2010) Cyclin B1 is a prognostic proliferation marker with a high reproducibility in a population-based lymph node negative breast cancer cohort. Int J Cancer 127: 961-967.

Ortiz-Martínez F, Perez-Balaguer A, Ciprián D, Andrés L, Ponce J, Adrover E, Sánchez-Payá J, Aranda FI, Lerma E, Peiró G (2014) Association of increased osteopontin and splice variant-c mRNA expression with HER2 and triple-negative/basal-like breast carcinomas subtypes and recurrence. Hum Pathol 45: 504-512.

Pang H, Lu H, Song H, Meng Q, Zhao Y, Liu N, Lan F, Liu Y, Yan S, Dong X, Cai L (2013) Prognostic values of osteopontin-c, E-cadherin and b-catenin in breast cancer. Cancer Epidemiol 37: 985-992.

Patani N, Jiang W, Mokbel K (2008a) Osteopontin C mRNA expression is associated with a poor clinical outcome in human breast cancer. Int $J$ Cancer 122: 2646.

Patani N, Jouhra F, Jiang W, Mokbel K (2008b) Osteopontin expression profiles predict pathological and clinical outcome in breast cancer. Anticancer Res 28: 4105-4110.

Rudland PS, Platt-Higgins A, El-Tanani M, De Silva Rudland S, Barraclough R, Winstanley JH, Howitt R, West CR (2002) Prognostic significance of the metastasis-associated protein osteopontin in human breast cancer. Cancer Res 62: 3417-3427.

Senger DR, Peruzzi CA, Papadopulous A, Tenen DG (1989) Purification of a human milk protein closely similar to tumor-secreted phosphoproteins and osteopontin. Biochim Biophys Acta 996: 43-48.

Sullivan J, Blair L, Alnajar A, Aziz T, Ng CY, Chipitsyna G, Gong Q, Witkiewicz W, Weber GF, Yeo CJ, Arafat HA (2009) Expression of a prometastatic splice variant of Osteopontin, OPNc, in human pancreatic ductal adenocarcinoma. Surgery 146: 232-240.

Sullivan J, Blair L, Alnajar A, Aziz T, Chipitsyna G, Gong Q, Yeo CJ, Arafat HA (2011) Expression and regulation of nicotine receptor and osteopontin isoforms in human pancreatic ductal adenocarcinoma. Histol Histopathol 26: 893-904.

Tilli TM, Franco VF, Robbs BK, Wanderley JL, Silva F, Duarte de Mello K, Viola JPB, Weber GF, Gimba ERP (2011) Osteopontin-c splicing isoform contributes to ovarian cancer progression. Mol Cancer Res 9: 280-293.

Tilli TM, Thuler LCS, Matos AR, Coutinho-Camillo CM, Soares FA, da Silva EA, Neves AF, Goulart LR, Gimba ER (2012) Expression analysis 
of osteopontin mRNA splice variants in prostate cancer and benign prostatic hyperplasia. Exp Mol Pathol 92: 13-19.

Tuck AB, O’Malley FP, Singhal H, Harris JF, Tonkin KS, Kerkvliet N, Saad Z, Doig GS, Chambers AF (1998) Osteopontin expression in a group of lymph node negative breast cancer patients. Int J Cancer 79: 502-508.

Wang X, Chao L, Ma G, Chen L, Tian B, Zang Y, Sun J (2008) Increased expression of osteopontin in patients with triple-negative breast cancer. Eur J Clin Invest 38: 438-446.

Weber GF, Lett S, Haubein N (2010) Osteopontin is a marker for cancer aggressiveness and patient survival. Br J Cancer 103: 861-869.

Weber GF, Lett GS, Haubein NC (2011) Meta-analysis of Osteopontin as a clinical cancer marker. Oncol Rep 25: 433-441.
Weber GF (2011) The cancer biomarker Osteopontin: combination with other markers. Cancer Genomics Proteomics 8: 263-288.

Zhang M-X, Xu Y-J, Zhu M-C, Yan F (2013) Overexpressed ostepontin-c as a potential biomarker for esophageal squamous cell carcinoma. Asian Pac $J$ Cancer Prev 14: 7315-7319.

This work is published under the standard license to publish agreement. After 12 months the work will become freely available and the license terms will switch to a Creative Commons AttributionNonCommercial-Share Alike 4.0 Unported License. 